\title{
Effects of elevated calcium on motor and exploratory activities of rats
}

A.F. Godinho,

T.V. Trombini and

E.C. Oliveira
Centro de Assistência Toxicológica (CEATOX), Instituto de Biociências,

Universidade Estadual Paulista, Botucatu, SP, Brasil

\author{
Correspondence \\ A.F. Godinho \\ CEATOX, Instituto de Biociências \\ UNESP \\ Rubião Junior, $s / n$ \\ Caixa Postal 523 \\ 18618-000 Botucatu, SP \\ Brasil \\ Tel/Fax: + 55-14-6821-3048 \\ E-mail: godinho@ibb.unesp.br
}

Publication supported by FAPESP.

Received February 14, 2001 Accepted February 20, 2002

\section{Abstract}

The effects of serum and brain calcium concentration on rat behavior were tested by maintaining animals on either distilled water $(\mathrm{N}=60)$ or water containing $1 \%$ calcium gluconate $(\mathrm{N}=60)$ for 3 days. Animals that were maintained on high calcium drinking water presented increased serum calcium levels (control $=10.12 \pm 0.46 v s$ calcium treated $=11.62 \pm 0.51 \mu \mathrm{g} / \mathrm{dl}$ ). Increase of brain calcium levels was not statistically significant. In the behavioral experiments each rat was used for only one test. Rats that were maintained on high calcium drinking water showed increased open-field behavior of ambulation (20.68\%) and rearing (64.57\%). On the hole-board, calcium-supplemented animals showed increased head-dip (67\%) and head-dipping (126\%), suggesting increased ambulatory and exploratory behavior. The time of social interaction was normal in animals maintained on drinking water containing added calcium. Rats supplemented with calcium and submitted to elevated plus-maze tests showed a normal status of anxiety and elevated locomotor activity. We conclude that elevated levels of calcium enhance motor and exploratory behavior of rats without inducing other behavioral alterations. These data suggest the need for a more detailed analysis of several current proposals for the use of calcium therapy in humans, for example in altered blood pressure states, bone mineral metabolism disorders in the elderly, hypocalcemic states, and athletic activities.

\section{Introduction}

Calcium is an essential biological regulator which influences a large number of fundamental intracellular processes $(1,2)$. A variety of studies have been performed to investigate alterations in calcium levels associated with pathological conditions. Transient increases in serum calcium and inorganic phosphorus levels have been observed during periodically recurrent, abrupt, spontaneous exacerbation of psychotic agitation and mania $(3,4)$. In contrast, the reduction of psychotic agitation or mania by the hypocalcemic and hypophosphatemic hormone calcitonin supports the view that the transient increase in serum calcium and phosphorus levels which accompanies exacerbation of acute psychotic agitation $(3,4)$ may play a causal role in such abrupt excitation (5).

It has been shown that increases in serum calcium levels through intravenous infusion of calcium lactate in a patient with paranoid psychosis secondary to hypoparathyroidism repeatedly provoke exacerbation of patient psychosis (6). Recently, it has been observed 
that increased serum calcium related to mania or excited states and the appearance of mania followed calcium replacement $(7,8)$.

Most of these studies measured serum calcium levels, which do not necessarily reflect tissue calcium levels. However, since these studies were purely correlational in nature, they did not determine whether the altered calcium levels were a cause or a consequence of the disease state.

Considerable attention is being centered on therapeutic benefits derived from dietary calcium supplementation, for example in altered blood pressure states (9-11), bone mineral metabolism disorders in the elderly (12$14)$, and in hypocalcemic states $(15,16)$. Surprisingly, little effort has been devoted to studies on the possible side effects of such therapy since a variety of cellular mediators of the cellular events involved in cell physiology and pathology are activated by an increase in intracellular free calcium $(1,2)$.

It has been suggested that increased serum calcium alters calcium levels in the cerebrospinal fluid and leads to altered excitability of the central nervous system (17). Furthermore, a reduction in the effect of amphetamine-induced motor activities by calcium channel blockers was also observed (18). Drinking water containing elevated calcium levels has been reported to elevate levels of brain $(2.43$ before and $2.88 \mathrm{mM}$ after) and serum ( 8.14 before and $10.10 \mathrm{mM}$ after) calcium associated with enhanced learned helplessness and decreased brain serotonin turnover (19).

These observations suggest that more attention should be paid to studies on the possible side effects of dietary calcium supplementation therapy. Since the evidence that calcium supplementation therapy leads to acute alterations in calcium levels (19) and that alterations of calcium levels would produce behavioral alterations in humans and laboratory animals, the objective of this study was to determine whether an acute elevation of calcium ingestion through the addition of $1 \%$ calcium gluconate to drinking water for 3 days can alter serum and brain calcium levels and behavior of rats.

\section{Material and Methods}

\section{Animals and treatment}

Male Wistar rats weighing 150-160 g were kept under standard conditions (up to five rats/cage, $25 \pm 2{ }^{\circ} \mathrm{C}, 70 \%$ humidity, 12 -h light-dark cycle, with lights on at 6:00 am, Purina rat chow and water ad libitum) for 2 weeks prior to the test procedure. Animals were randomly assigned to two groups of 60 rats each. Group I was maintained for 3 days on distilled water containing $1 \%(\mathrm{w} / \mathrm{v})$ calcium gluconate. Group II (control group) was maintained for 3 days on distilled water. During the treatment period, food and water ingestion were monitored at 24-h intervals and animals were observed continuously.

\section{Behavioral tests}

At the end of a 72-h period of exposure to calcium or distilled water, groups of rats were submitted to open-field, social interaction, hole-board, and elevated plus-maze tests. The tests were conducted in a soundattenuated, temperature-controlled room illuminated by dim red light. Rats were used for only one test.

\section{Open-field}

Open-field behavior was assessed using a wood box measuring $97 \times 32.5 \mathrm{~cm}$ (diameter $x$ height), similar to that described in Ref. 20. The box was divided into three concentric circles, which were subdivided by black painted lines into 18 similar spaces. For open-field observations, each rat was individually placed in the center of the arena and the following parameters were scored for $3 \mathrm{~min}$ : ambulation frequency (number of floor units entered with the paws), rearing 
frequency (number of times the animal stood on its hind legs), freezing duration (total time the animal was in an immobile state, often in a crouching posture with wide-open eyes and irregular respiration), and grooming duration (total time the animal used to groom). The following grooming behaviors were considered: forepaw vibration; paw licking; washing of nose, face and head; body licking; genital grooming; scratching, and head-shaking. The open-field was washed with $5 \%$ ethanol before a new animal was introduced.

\section{Social interaction}

All observations for the social interaction test were carried out in the open-field arena. The social interaction test, which is sensitive to anxiogenic drugs (21), consisted of familiarizing each pair (cage mates) of rats with the arena for a period of $8 \mathrm{~min}$ on two consecutive days. On the third day, each rat was assigned to an unfamiliar partner according to weight and placed in the test arena for observation of social interaction behavior and overall activity for $5 \mathrm{~min}$. Social interaction time (in seconds) per pair of rats was measured as the time spent sniffing the partner, climbing over and crawling under the partner, mutual grooming, genital investigation, and following and walking around the partner (21). Aggressive behavior was not considered to be social interaction behavior.

\section{Hole-board}

The hole-board test was an open-field arena with four equally spaced holes $(3 \mathrm{~cm}$ in diameter) in the floor, as described by File and Wardill (22). In the hole-board, head-dip (count of the number of times the animal inserts the head into the hole) and head-dipping (duration of head-dip in seconds) were recorded for 5 min, with a head-dip scored if both eyes disappeared into the hole.

\section{Elevated plus-maze}

The elevated plus-maze test was conducted as described in Ref. 23 and assessed using an apparatus consisting of two open and two enclosed arms of equal length and width $(50 \times 10 \mathrm{~cm})$. The open arms had a 1-cm high Plexiglas edge while the enclosed arms had $40-\mathrm{cm}$ high wooden sides. The plus-maze was elevated $50 \mathrm{~cm}$ above the floor. Each rat was placed in the center of the elevated plus-maze facing one of the open arms, with the number of entries with the four paws and time spent (seconds) in the open or closed arms recorded during a 3-min test period. The elevated plus-maze test is based on the principle that exposure to an elevated and open arm maze leads to an approach conflict that is considerably stronger than that evoked by exposure to an enclosed maze arm. Thus, the relation between total entries and time spent in open and closed arms provides a measure of anxiety or fear-induced inhibition of normal exploratory activity (23). The calculated anxiety index is obtained by dividing the number of entries into open arms by the number of entries into open plus closed arms. Another parameter obtained with the elevated plusmaze is the latency time to animal entry with all four paws into closed arms when put in the extremity of the open arm. This test has been proposed to the study of memory and is influenced by the motor activity status of the animals (24). For this test, animals were previously familiarized with the elevated plus-maze for a period of $5 \mathrm{~min}$ on three consecutive days.

The elevated plus-maze apparatus was carefully washed with $5 \%$ ethanol before a new animal was introduced for testing.

\section{Blood and brain calcium evaluation}

After the behavioral tests, rats selected at random were anesthetized (pentobarbital, $i p$ ) and blood was collected by heart puncture. 
Animals were killed by decapitation and brains were removed, weighed, and homogenized using a Potter apparatus. Analysis of calcium was performed using a Hitachi (model Z-5700) atomic absorption spectrophotometer graphite-furnace atomizer with adaptations (25).

Table 1. Effect of treatment with calcium gluconate on serum and brain calcium levels of rats.

\begin{tabular}{lcc}
\hline Group & Serum $(\mu \mathrm{g} / \mathrm{dl})$ & Brain $(\mu \mathrm{g} / \mathrm{g}$ wet weight) \\
\hline Control & $10.12 \pm 0.46$ & $98.70 \pm 7.90$ \\
Calcium gluconate & $11.62 \pm 0.51^{*}$ & $113.50 \pm 8.10$
\end{tabular}

Data are reported as means \pm SEM $(\mathrm{N}=10) .{ }^{*} \mathrm{P}<0.05$ compared to control (Student t-test).

Table 2. Open-field and hole-board behavior of rats receiving distilled water containing $1 \%$ calcium gluconate for 3 days.

\begin{tabular}{lrc}
\hline Test & \multicolumn{1}{c}{ Control } & Calcium gluconate \\
\hline Open-field & & \\
$\quad$ Ambulation (count) & $63.60 \pm 3.54$ & $76.75 \pm 4.21^{*}$ \\
Rearing (count) & $26.73 \pm 2.33$ & $43.99 \pm 2.54^{*}$ \\
Freezing (s) & $1.50 \pm 0.36$ & $1.56 \pm 0.72$ \\
$\quad$ Grooming (s) & $13.32 \pm 2.07$ & $11.63 \pm 1.72$ \\
Hole-board & & \\
$\quad$ Head-dip (count) & $7.25 \pm 0.85$ & $12.15 \pm 1.95^{*}$ \\
Head-dipping (s) & $8.55 \pm 1.21$ & $19.35 \pm 1.77^{*}$ \\
\hline
\end{tabular}

Data are reported as means \pm SEM $(\mathrm{N}=15) . * \mathrm{P}<0.05$ compared to control (Student t-test).

Table 3. Elevated plus-maze behavior of rats receiving distilled water containing $1 \%$ calcium gluconate for 3 days.

\begin{tabular}{lcc}
\hline Test & Control & Calcium gluconate \\
\hline Open arm entries (count) & $5.10 \pm 0.71$ & $9.70 \pm 1.45^{*}$ \\
Closed arm entries (count) & $4.00 \pm 0.46$ & $7.60 \pm 1.06^{*}$ \\
Time in open arm (s) & $89.20 \pm 13.38$ & $76.30 \pm 12.93$ \\
Time in closed arm (s) & $124.40 \pm 22.66$ & $106.00 \pm 14.70$ \\
Anxiety index & $0.56 \pm 0.09$ & $0.56 \pm 0.08$ \\
Latency time (s) & $5.40 \pm 0.41$ & $4.09 \pm 0.35^{*}$
\end{tabular}

Anxiety index $=$ entries into open arms/entries into open plus closed arms. Latency time $=$ time for animal to enter with all four paws into the closed arms when placed at the entrance of the open arm. Data are reported as means \pm SEM $(\mathrm{N}=15) . * \mathrm{P}<0.05$ compared to control (Student t-test).

\section{Statistical analysis}

The two-way Student $t$-test was used for comparison between the control and experimental groups (26). Differences between groups were considered significant when $\mathrm{P}<0.05$.

\section{Results}

During the treatment period food or water ingestion was not changed by calcium supplementation (data not shown). Table 1 shows that calcium levels were significantly increased in the serum $[t(18)=2.184, \mathrm{P}=$ 0.0424 ] of calcium-supplemented animals by $15 \%$ of the mean; however, for brains the observed increase was not statistically significant $[t(18)=1.308, \mathrm{P}=0.2073]$.

Table 2 shows that the addition of $1 \%$ calcium gluconate to the drinking water of the rats for 3 days increased the open-field behaviors of ambulation $[t(28)=2.391, \mathrm{P}=$ $0.0238]$ and rearing $[t(28)=5.008, \mathrm{P}=$ $0.0001]$. The freezing $[t(28)=0.745, \mathrm{P}=$ $0.9411]$ or grooming $[t(28)=0.6279, \mathrm{P}=$ $0.5351]$ behavior was not altered by calcium supplementation. The data for hole-board experimentation (Table 2) show a significant increase in head-dip $[t(28)=2.303, \mathrm{P}=$ $0.0289]$ and head-dipping $[t(28)=5.037, \mathrm{P}=$ 0.0001 ] behaviors, suggesting enhanced exploratory behavior in calcium-supplemented animals.

Supplementation with calcium gluconate did not alter $[t(28)=0.1446, \mathrm{P}=0.8861]$ the social interaction behavior of the rats who presented interaction times of $53.07 \pm 6.25$ and $55.30 \pm 6.77$ for control $(\mathrm{N}=15)$ and calcium-supplemented $(\mathrm{N}=15)$ rats, respectively.

The data in Table 3 show that the calcium-supplemented animals, when submitted to elevated plus-maze experimentation, presented a significant increase in open $[t(28)$ $=2.849, \mathrm{P}=0.0081]$ and closed $[t(28)=$ $3.116, \mathrm{P}=0.0042]$ arm entries and a lower 
time of permanence in both open $[t(28)=$ $0.6933, \mathrm{P}=0.4938]$ and closed $[t(28)=$ $0.6812, \mathrm{P}=0.5013$ ] arms. Although these results show an apparent preference of the animals for the open arms of the elevated plus-maze, the anxiety index calculated for animals receiving calcium supplementation was equal to that calculated for the control $[t(28)=0.000, \mathrm{P}=1.0000]$.

In the other group tested in the elevated plus-maze, the animals receiving calcium supplementation presented a significant decrease $[t(28)=2.430, \mathrm{P}=0.0218]$, in relation to controls, in the latency time to enter the closed arms when placed at the extremity of the open arm (Table 3).

To study the possibility of treatment modifying habituation of the animals to the test apparatus, we performed an experiment using one group of naive rats only receiving calcium gluconate and another receiving calcium gluconate and habituated to the elevated plus-maze ( $5 \mathrm{~min}$ for three consecutive days). The observed latency time was equal for these two groups $[t(28)=0.8724, \mathrm{P}$ $=0.3904]$, showing that the treatment did not modify the habituation status of the animals (data not shown).

\section{Discussion}

The present data suggest that animals maintained on drinking water containing calcium supplement may undergo a critical stimulatory effect on motor or exploratory behavior. Rats maintained on drinking water supplemented with enough calcium gluconate to statistically increase plasma calcium levels by approximately $15 \%$, but not brain levels, greatly enhanced motor or exploratory activity response.

Drinking water containing elevated calcium levels has been reported to elevate levels of serum and brain calcium associated with altered behavior and neurotransmitter turnover (19). The present data agree with these previous observations of serum and brain calcium elevation after calcium supplementation of drinking water. The differences in percent increase are probably due to the time of treatment and calcium salt utilized.

Our results with measurement of serum and brain calcium do not permit us to predict if an increase of calcium occurs in the motor plate, in the central nervous system, or both. Taken together, our data seem to indicate alterations mainly provoked by peripheral effects. Perhaps an experiment performed with administration of higher concentrations of calcium would clarify this question; however, administration of high calcium concentrations might provoke alterations at various levels of the organism including neurotransmitter metabolism and activity (19), and indirectly influence animal behavior.

In our experiments, following calcium supplementation a significant increase in ambulation and rearing was observed in openfield. As reported by Satinder (27), rearing and ambulation are positively correlated behaviors. The observed changes in locomotor activity and rearing in calcium-supplemented rats are in agreement with this premise. This stimulant motor effect observed in our experiments is of a magnitude comparable to that obtained by White and Blackburn (28) with administration of a dose of $1 \mathrm{mg} / \mathrm{kg}$ of d-amphetamine. In contrast, previous data showed that calcium channel blockers inhibited increased amphetamine $(0.6 \mathrm{mg} / \mathrm{kg})$ induced motor activity (18).

Furthermore, since the association of ambulation with rearing can be regarded as an exploratory behavior (29), another approach was to investigate the ability of the calciumsupplemented animals to explore the holeboard, a valid test of exploration (22). In agreement with the results obtained from animals submitted to the open-field, increased exploratory behavior was observed in the hole-board, which shows that the exploratory ability of rats is positively influenced by calcium supplementation. Since ambula- 
tion is an inverse measure of emotionality (29), the observed increase in ambulation and rearing might be the consequence of an altered emotional state of animals due to elevated calcium levels.

These concerns were completely resolved using the elevated plus-maze and the social interaction tests, which are two models of rodent anxiety used extensively in the discovery of novel anxiolytic and anxiogenic agents and also to investigate the psychological and neurochemical basis of anxiety $(21,23,30,31)$.

The numbers of entries into the open and closed arms obtained in the elevated plusmaze for calcium-supplemented rats were higher than those obtained for the controls, probably because of the influence of treatment on motor or exploratory behavior of animals. The fact that calcium-supplemented rats remain less time in both the open and closed arms suggests a higher motor-stimulant effect provoked by treatment. The anxiety index and the duration of social interaction were not modified following calcium supplementation, suggesting no alterations in the emotional state of the animals.

The latency time for animal entry into closed arms when placed on the extremity of the open arm of the elevated plus-maze was statistically lower for animals maintained on drinking water containing calcium gluconate. Since the animals were acquainted with the elevated plus-maze environment and had normal emotional status, these data support the suggestion of a motor-stimulant effect caused by high calcium levels.

Apparently, calcium-supplemented rats behave as if they had received a non-anxiolytic agent acting on the central nervous system and the increased exploratory behavior is probably due to the motor-stimulant effect provoked by an enhanced calcium level.

Since elevated calcium levels alter neurotransmitter metabolism and activity (19), we cannot be certain if the behavioral effects observed are due solely to enhanced calcium levels. Thus, alterations at the neurotransmitter level need to be studied.

The present data demonstrate that administration of $1 \%$ calcium gluconate in the drinking water for 3 days increased the motor or exploratory behavior of rats without inducing other behavioral alterations. Since a transient increase in total serum calcium concentration can be accompanied by functional alterations in different systems of the organism, including the central and peripheral nervous systems, and the onset of mania or other excitatory states can be related to transient altered calcium levels, we suggest that more attention should be paid to calcium salt supplements in humans.

\section{Acknowledgments}

The authors thank James R. Welsh and Thiago Rocha A. Duarte for excellent technical assistance.

\section{References}

1. Campbell AK (1987). Intracellular calcium: friend or foe? Clinical Science, 72: 1-10.

2. Carafoli E, Caroni P, Chiesi M \& Famulski $\mathrm{K}$ (1982). $\mathrm{Ca}^{2+}$ as a metabolic regulator: Mechanisms for the control of its intracelIular activity. In: Sies H (Editor), Metabolic Compartmentation. Academic Press, New York, NY, USA, 521-547.

3. Carman J S \& Wyatt RJ (1977). Alterations in CSFG and serum total calcium with changes in psychiatric state. In: Usdin E,
Hamburg DA \& Bachas JD (Editors), Neuroregulators and Psychiatric Disorders. Oxford University Press, New York, NY, USA, 488-494.

4. Fischback R (1971). Changes in calcium metabolism in depression and during medication with thymoleptics. Arzneimittel-Forschung, 21: 27-28.

5. Carman J S \& Wyatt RJ (1979). Use of calcitonin in psychotic agitation or mania. Archives of General Psychiatry, 36: 72-75.
6. Snowdon JA, MacFie AC \& Pearce JB (1976). Hypocalcemic myopathy with paranoid psychosis. J ournal of Neurology, Neurosurgery and Psychiatry, 39: 48-52.

7. Carman J S, Post RM \& Runkle DC (1979). Increased serum calcium and phosphorus with the "switch" into mania or excited states. British J ournal of Psychiatry, 135: 55-61.

8. Groat RD \& MacKenzie TB (1980). The appearance of mania following calcium 
replacement. J ournal of Nervous and Mental Disease, 168: 562-563.

9. Ayachi S (1979). Increased dietary calcium lowers blood pressure in the spontaneously hypertensive rat. Metabolism, 28: 1234-1238.

10. McCarron DA, Morris CD \& Cole C (1982). Dietary calcium in human hypertension. Science, 217: 267-269.

11. Morris CD \& McCarron DA (1992). Effect of calcium supplementation in an older population with mildly increased blood pressure. American J oumal of Hypertension, 5: 230-237.

12. Dawson-Hughes B, Harris SS, Krall EA \& Dallal GE (1997). Effect of calcium and vitamin $D$ supplementation on bone density in men and women 65 years of age or older. New England J ournal of Medicine, 337: 670-676.

13. Goddard M, Young G \& Marais R (1986). Short-term effects of calcium carbonate, lactate, and gluconate on the calciumparathyroid axis in normal elderly men and women. American J ournal of Clinical Nutrition, 44: 653-658.

14. Rourke K, Bowering J , Turkki P, Buckenmeyer O, Keller B \& Sforzo G (1998). Effect of calcium supplementation on bone mineral density in female athletes. Nutrition Research, 18: 775-783.

15. Carroli G, Duley L, Belizan J M \& Villar J (1994). Calcium supplementation during pregnancy: a systematic review of randomised controlled trials. British J ournal of Obstetrics and Gynaecology, 101: 753758.
16. Palmer S (1982). Disorders of calcium metabolism in children. In: Anghileri LJ \& Anghileri AMT (Editors), The Role of Calcium in Biological Systems. Vol. III. CRC Press Inc., Boca Raton, FL, USA.

17. Veale WL \& Myers RD (1971). Emotional behavior, arousal and sleep produced by sodium and calcium ions perfused within the hypothalamus of the cat. Physiology and Behavior, 7: 601-607.

18. Ansah TA, Wade LH \& Shockley DC (1993). Effects of calcium channel blockers on cocaine and amphetamineinduced motor activities and toxicities. Life Sciences, 53: 1947-1956.

19. Trulson ME, Arasteh K \& Ray DW (1986). Effects of elevated calcium on learned helplessness and brain serotonin metabolism in rats. Pharmacology, Biochemistry, and Behavior, 24: 445-448.

20. Broadhurst PL (1960). Experiments in psychogenetics. In: Eysenk HJ (Editor), Experiments in Personality. Vol. 1. Rutledge and Kegan Paul, London, UK.

21. Dunn RW, Corbett R \& Fielding S (1989). Effects of $5-\mathrm{HT}_{1 \mathrm{~A}}$ receptor agonists and NMDA receptor antagonists in the social interaction test and the elevated plus maze. European J ournal of Pharmacology, 169: 1-10.

22. File SE \& Wardill AG (1975). Validity of head-dipping as a measure of exploration in a modified holeboard. Psychopharmacology, 44: 53-59.

23. Pellow S \& File SE (1986). Anxiolytic and anxiogenic drug effects on exploratory activity in an elevated plus-maze: a novel test of anxiety in the rat. Pharmacology, Biochemistry, and Behavior, 24: 525-529.

24. Itoh J, Nabeshima $T \&$ Kameyama $T$ (1990). Utility of an elevated plus-maze for the evaluation of memory in mice: effects of nootropics, scopolamine and electroconvulsive schock. Psychopharmacology, 101: 27-33.

25. Yeger DW, Cholak J \& Henderson EW (1971). Determination of lead in biological and related material by atomic absorption spectrophotometry. Environmental Science and Technology, 5: 1020-1022.

26. Snedecor GW \& Cochran WG (1980). Statistical Methods. 7th edn. Iowa State University Press, Ames, IA, USA.

27. Satinder KP (1968). A note on the correlation between open-field and escape avoidance behavior in the rat. J ournal of Psychology, 69: 3-6.

28. White NM \& Blackburn J (1986). Effect of glucose on amphetamine-induced motor behavior. Life Sciences, 38: 2255-2262.

29. Archer J (1973). Tests for emotionality in rats and mice: a review. Animal Behaviour, 21: 205-235.

30. Dawson GE \& Tricklebank D (1995). Use of the elevated plus maze in the search for novel anxiolytic agents. Trends in Pharmacological Sciences, 16: 33-36.

31. File SE, Pellow S \& Braestrup C (1985). Effects of the ß-carboline, FG 7142, in the social interaction test of anxiety and the holeboard: correlation between behaviour and plasma concentrations. Pharmacology, Biochemistry, and Behavior, 22: 941944. 